\title{
Hydrocarbon-Derived Carbonate Cements of Subsurface Origin in the Vulcan Sub-Basin, Timor Sea
}

\author{
Shou-Yeh Gong ${ }^{1,}$, , Horng-Sheng Mii ${ }^{2}$, Ming-Shyan $\mathrm{Wu}^{3}$, Kuo-An Lin ${ }^{3}$, Shih-Wei Wang ${ }^{1}$, \\ Ting-Fang $\mathrm{Chou}^{3}$, Ying-Wei Chou ${ }^{3}$, Jong-Chang $\mathrm{Wu}^{3}$, and Yuh-Ruey Wang ${ }^{4}$ \\ ${ }^{1}$ Department of Geology, National Museum of Natural Science, Taichung, Taiwan \\ ${ }^{2}$ Department of Earth Sciences, National Taiwan Normal University, Taipei, Taiwan \\ ${ }^{3}$ Exploration and Development Research Institute, Chinese Petroleum Corporation, Miaoli, Taiwan \\ ${ }^{4}$ Department of Materials and Mineral Resources Engineering, National Taipei University of Technology, \\ Taipei, Taiwan
}

Received 7 September 2009, accepted 14 January 2010

\begin{abstract}
Localized carbonate cementation occurs in the Eocene Grebe Sandstone of the Vulcan Sub-basin, Timor Sea, Australia. The cements have been previously interpreted as originating from microbial methane oxidation and sulfate reduction in a shallow subsurface environment and were related to hydrocarbon leakage. Here we reassess these localized carbonate cements in the Grebe Sandstone, and reported new findings. Petrography shows that there are two facies of sands in the Grebe Sandstone: (1) cemented, mostly fine-grained sands; and (2) loose, often coarse-grained sands. In addition, two types of carbonate matrix occur in the Grebe Sandstone: (1) spars to microspars in calcareous, fine-grained sandstones; and (2) micritic to microsparry matrix associated with limestone grains. Stable carbon isotopic values reveal that only the cements associated with sandstones were probably hydrocarbon-derived, and the resultant mineral is mainly calcite. Petrographic attributes and $\mathrm{Mn}^{+2}$ and $\mathrm{Co}^{+2}$ compositions of these cements differ significantly from those of modern cold-seep carbonates at or near the sea floor. Moreover, the hydrocarbon-derived carbonate mineralization only occurs in the fine-grained sands, not in the coarse-grained sands. In other word, the cementation was not only dependent on hydrocarbon leakage but also on the lithofacies of the host rock. We propose that the extent of hydrocarbon-related cementation alone cannot be used to evaluate the trap integrity as has been previously suggested.
\end{abstract}

Key words: Authigenic carbonate, Subsurface cementation, Hydrocarbon, Vulcan Sub-basin, Timor Sea

Citation: Gong, S. Y., H. S. Mii, M. S. Wu, K. A. Lin, S. W. Wang, T. F. Chou, Y. W. Chou, J. C. Wu, and Y. R. Wang, 2010: Hydrocarbon-derived carbonate cements of subsurface origin in the Vulcan Sub-basin, Timor Sea. Terr. Atmos. Ocean. Sci., 21, 923-937, doi: 10.3319/TAO.2010.01.14.01(TT)

\section{INTRODUCTION}

Researches have reported that localized carbonate cementation occurred in the Grebe Sandstone of the Vulcan Sub-basin, Timor Sea, which is Eocene in age and consists of mostly sandstones and some thin limestone beds (Figs. 1 and 2)(Woods 1991; O'Brien and Woods 1995). The localized cementation resulted in mound-like structures on seismic profiles, which were referred to as the Hydrocarbon Related Diagenetic Zone (HRDZ) and were interpreted as having resulted from microbial methane oxidation and sulfate reduction related to hydrocarbon leakage in shallow

\footnotetext{
* Corresponding author

E-mail:gng@mail.nmns.edu.tw
}

burial environment (O'Brien and Woods 1995; O'Brien et al. 1996, 1999; Cowley and O'Brien 2000; Gartrell et al. 2004; also see Fig. 3). The extent of the cemented zone was further considered to be a measurement of hydrocarbon leakage (O'Brien et al. 1998, 1999). The occurrence of such cementation thus may provide an important new tool in hydrocarbon exploration.

Occurrences of hydrocarbon-related carbonates in subsurface environments may also be of great academic interest. Authigenic carbonates have been found at many methane seeps and vents at or near the sea floor and generally interpreted to be microbial in origin (Paull et al. 1984; Hovland et al. 1987, 1994; Roberts et al. 1989; Aloisi et al. 
2000; Campbell 2006). However, the occurrence of hydrocarbon-derived carbonate mineralization has been rarely if ever identified in subsurface environments.

One of the criteria in identifying hydrocarbon origin of carbonate minerals is stable isotopic composition. Hydrocarbon-derived carbonates typically have depleted carbon isotopic values because hydrocarbons originated from organic matter, while the oxygen isotopic values are variable depending on the source of oxygen (Aharon 1994; Whiticar 1999; Campbell 2006). Trace elemental composition of hydrocarbon-related carbonates has not often been measured except a case in sea-floor cold-seep carbonate (Díaz-del-Río et al. 2003). This study presents the first results for trace elemental composition of hydrocarbon-related carbonates in a burial environment.

In this study, we reassessed these localized carbonate cements in the Grebe Sandstone. The objectives of this paper are: (1) to study the origin of localized carbonate cementation in the Grebe Sandstone; and (2) to understand controls of the distribution of these localized carbonate cements. We present results of petrographical, stable isotopic and elemental studies of cuttings from four exploration wells in the Vulcan Sub-basin, Timor Sea.

\section{REGIONAL SETTING}

The Vulcan Sub-basin is a NE/SW-trending basin, connecting to the Browse Basin to the southwest and the Bonaparte Basin to the northeast, in the Timor Sea, Australia (Fig. 1). Commercial oil discoveries have been made in the basin since 1983 (Cadman and Temple 2004). The sub-basin was formed by extension in the Jurassic, bounded by the Londonderry High in the southeast and the Ashmore Platform in the northwest (Patillo and Nicholls 1990; O'Brien et al. 1993). The convergence of the Australia plate and the Southeast Asian microplates during the Miocene and Pliocene reactivated the Jurassic extensional faults ( $\mathrm{Pa}$ tillo and Nicholls 1990; O'Brien et al. 1993).

The stratigraphy of the Vulcan Sub-basin is shown in Fig. 2. Thick carbonate sequences were deposited in the basin during the Cenozoic. In the Eocene and Oligocene lowstands, carbonate deposition was interrupted by deltaic sedimentation (Patillo and Nicholls 1990; O’Brien et al. 1993) The Eocene Grebe Sandstone is a lenticular sand body embedded between carbonate sequences of the Paleocene Johnson Formation and Eocene Hibernia Formation (Cadman and Temple 2004). According to O'Brien and Woods (1995) and O'Brien and others (1999), hydrocarbons in the Jurassic reservoirs leaked out and migrated along the faults into the Eocene Grebe Sandstone during the Miocene to Pliocene.

Localized carbonate cements commonly occurred in the Grebe Sandstones and can be identified on seismic profiles as indicated by time pull-up and amplitude anomaly

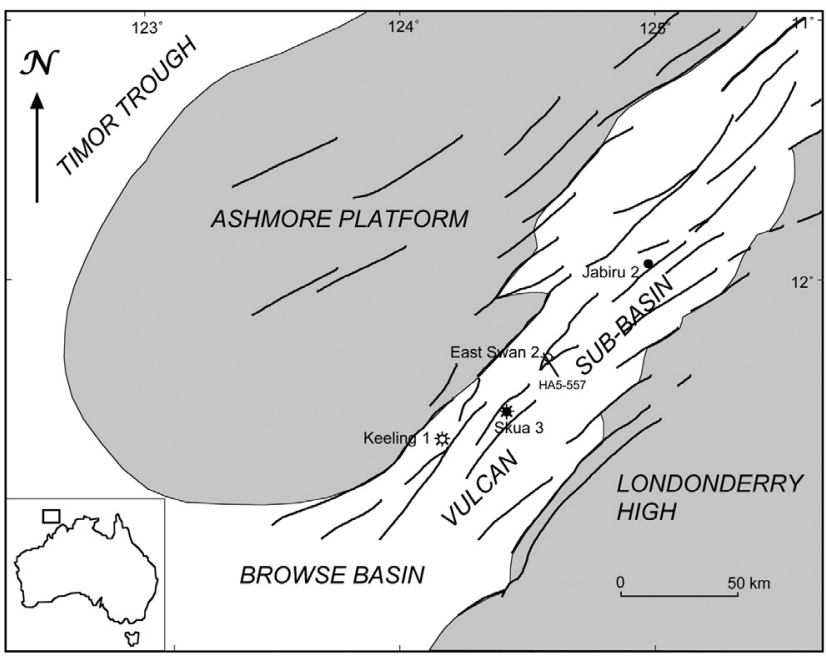

Fig. 1. Structural elements of Vulcan Sub-basin and the locations of study wells and seismic profile HA-5-557 (Fig. 3). Modified after Cadman and Temple (2004).

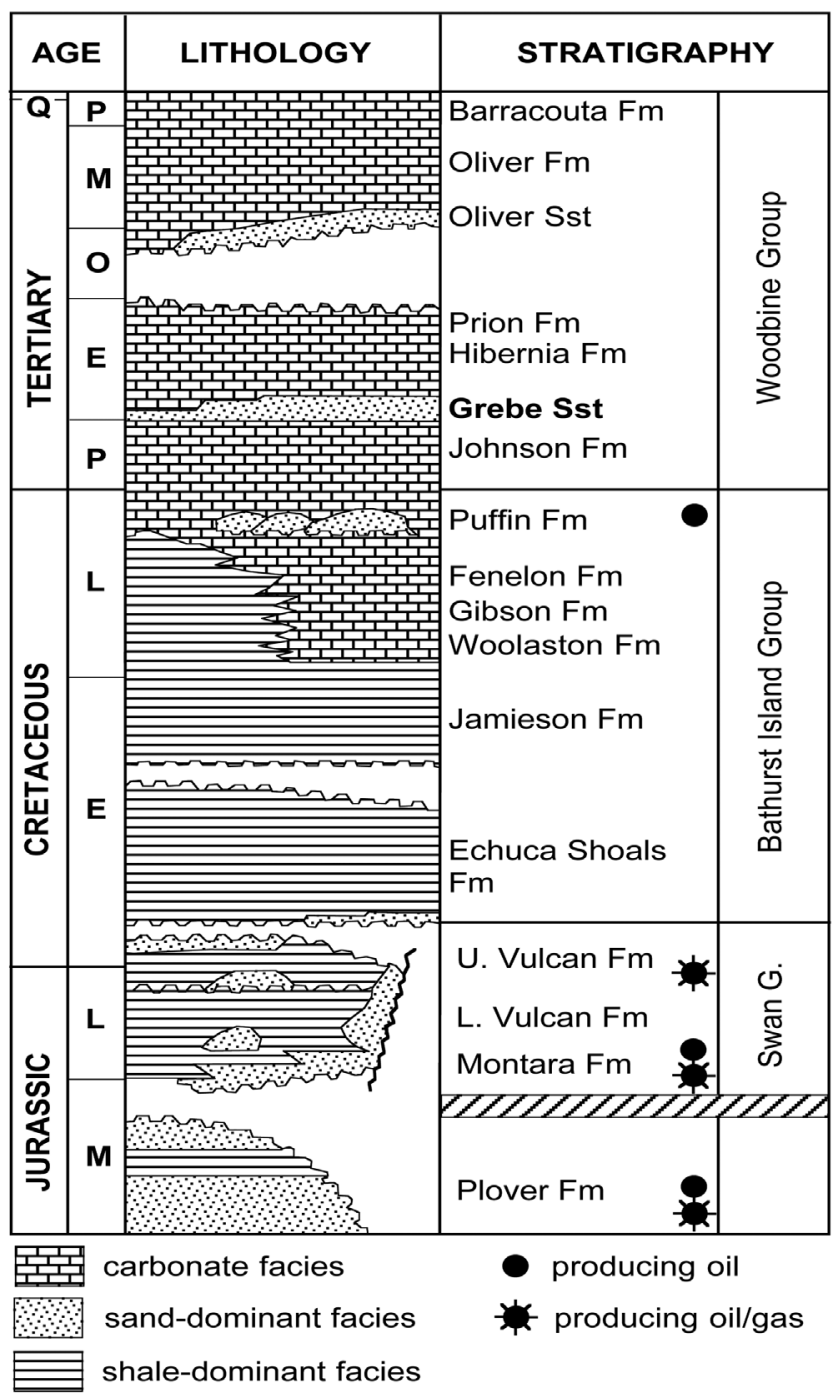

Fig. 2. Stratigraphy and lithology of the Vulcan Sub-basin. After Osborne (1990). 


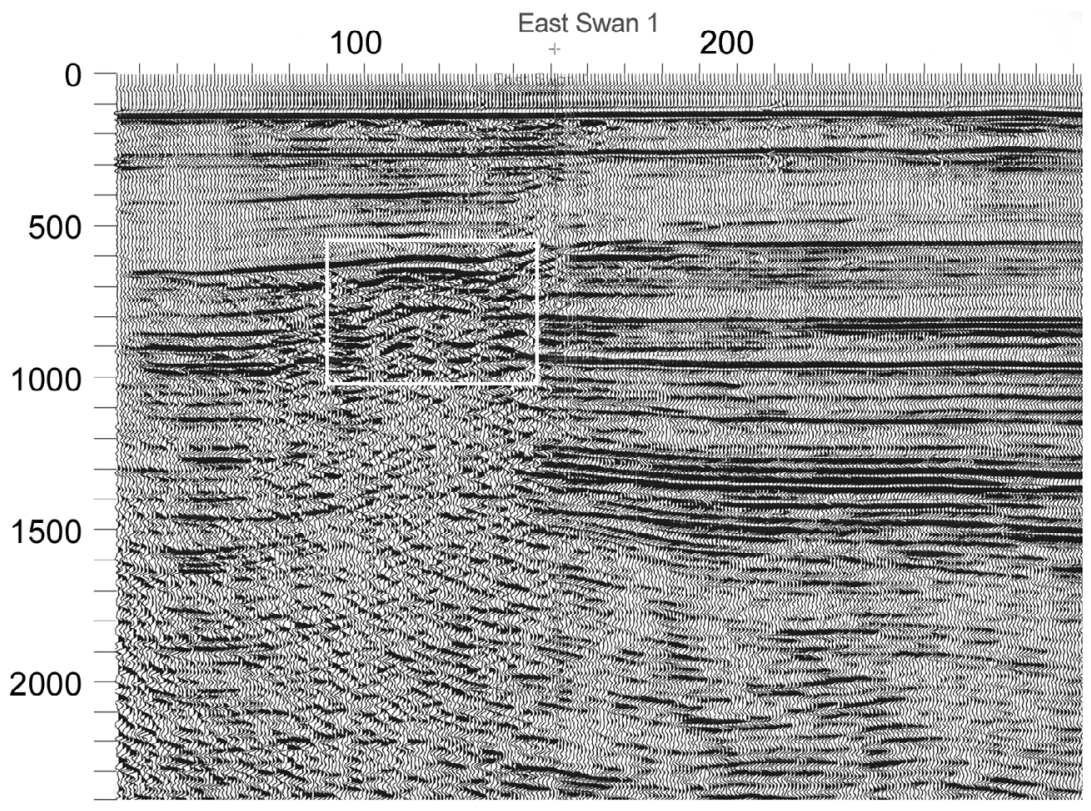

Fig. 3. Part of seismic line HA5-557, its location shown in Fig. 1, that passes nearby well East Swan-2. The vertical unit is two-way time in millisecond. Localized carbonate cementation is shown in rectangle and was confirmed by well East Swan-2 (O'Brien and Woods 1995).

marked in the rectangle of Fig. 3, and by comparing logs of adjacent wells (O’Brien and Woods 1995).

\section{MATERIALS AND METHODS}

We selected the following wells for this study: East Swan-2, Jabiru-2, Keeling-1, and Skua-3, based on the work of O'Brien and Woods (1995)(Fig. 1). Cutting samples, well logs and seismic profiles were acquired from the Australia National Petroleum Database via the Chinese Petroleum Corporation (Taiwan). In total, 115 cutting samples were included in this study.

A fraction of each sample was treated with dilute $\mathrm{HCl}$ to dissolve all the carbonate components, and weight percentages of carbonate and insoluble components were recorded. The percentages were compared to the sonic log to confirm the depth control of cuttings (Fig. 4). All the insoluble grains of each well that consist almost entirely of quartz were combined together and sieved to obtain their size distribution. The rest of cuttings from each depth interval were examined under a binocular microscope. Cuttings of calcareous sandstone and limestone grains - bioclastics excluded, were identified and handpicked for further studies; some large pieces were picked for thin-section or SEM studies. For each well except Skua-3, which contained too few calcareous sandstones, a portion of the calcareous sandstones picked from each depth interval was then combined together, treated with dilute $\mathrm{HCl}$ to remove the carbonates, and the quartz sand residues were then sieved to acquire their size distribution.

Due to the nature of well cuttings, calcareous sand- stone and limestone grains used for mineralogical and geochemical analysis were rinsed for about 1 second in $5 \% \mathrm{HCl}$ then washed in distilled water to remove possible stains on the surface. The mineralogy was determined using X-ray diffractometry (XRD). Stable carbon and oxygen isotopic compositions of the carbonates were analyzed using a Micromass IsoPrime mass spectrometer equipped with a multicarb automation system at National Taiwan Normal University, following the method of McCrea (1950). Analyses were performed on about $0.1 \mathrm{mg}$ of microsamples. $\mathrm{CO}_{2}$ was evolved from the carbonates reacted with $100 \%$ phosphoric acid at $90^{\circ} \mathrm{C}$ for 15 minutes. Results are reported in the $\delta$ per mil notation with respect to the V-PDB standard calibrated using international NBS-19 standard $\left(\delta^{13} \mathrm{C}=1.95 \%\right.$; $\delta^{18} \mathrm{O}=-2.20 \%$ ). The analytical precision was better than $\pm 0.05 \%$ \% $(1 \sigma, \mathrm{N}=591)$ for both carbon and oxygen isotopes. Some samples were measured twice to test data reproducibility (Table 1). Manganese and cobalt elemental analyses of the carbonate cements were performed using a PerkinElmer Optima 2000 ICP-AES at the National Museum of Natural Science. Proportional calcium and magnesium ions were used with manganese and cobalt ions in standard solutions to account for possible matrix effects. The regression coefficient of the standards was maintained at 0.9995 or better and Relative Standard Deviation at $1 \%$ or better for all analyses.

\section{PETROGRAPHY AND MINERALOGY}

Two types of sandstones can be distinguished in the Grebe Sandstone: (1) calcareous cemented sandstones that 
are mostly fined-grained, relatively immature in angularity; and (2) poorly cemented sands that are often coarse-grained and well rounded but also include some fine sands (Figs. 5 and 6). The quartz grains in the calcareous sandstones typically have an angular to subangular shape while those comprising the coarse-grained loose sands are mostly well rounded.

We could not measure the size distribution of the loose sands because of the difficulties in hand-picking all the loose sands from the calcareous sandstone, also because of the relative abundance of loose sands and cemented sandstones varying from depth interval to depth interval. Instead, the size distribution of the total quartz sands, including both of the cemented and loose sands, was measured. Figure 5 shows that the quartz grains of the calcareous sandstones are significantly smaller in grain-size compared to the total sands. Please note that the total sands include loose sands and cemented sands, hence their sorting and grain-size distribution are "contaminated" by cemented sands. The loose sands should exhibit better sorting and coarser size than the total sands (Fig. 5).

We also observed two types of carbonate cements in the Grebe Sandstone under microscope: (1) white to white gray, sparry to microsparry cements associated with the calcareous sandstones; (2) light tan to light gray, micritic to microsparry matrix in limestone (Figs. 6a, c, and 7d). Toward the upper part of the Grebe Sandstone and into the Hibernia Formation, the cements appear light tan and are often associated with bioclastics, mostly larger foraminifera, while toward the lower part of the Grebe Sandstone and into the Johnson Formation the micrites are light gray and contain no bioclastics.

We did not observe any rock types or fossils that are typical of a methane-seep community, such as vesicomyids or lucinids, or fragments of dark gray, argillaceous, finegrained matrix/cement with breccias or plankton foraminifera (Hovland et al. 1987; Terzi et al. 1994; Aloisi et al. 2000; Greinert et al. 2001; Peckmann et al. 2001; Campbell et al. 2002; Wang et al. 2006).

XRD revealed that both calcite and dolomite are present in the sandstone cements and the limestone matrix. The relative abundance of the two minerals varies from sample to sample (Table 1). Aragonite was not identified by XRD, nor were aragonite needles observed under the microscope or SEM.

Given the nature of cutting samples, it is possible that the limestone cuttings found in the depth range of the Grebe Sandstone actually came from the overlying Hibernia and underlying Johnson Formations and mixed into the cuttings of Grebe Sandstone. However, the Grebe Sandstone occurs as a lenticular body embedded between the Johnson and Hibernia Formations. The well logs indicated that the Grebe Sandstone does include some thin limestone beds (Fig. 4). Some sandstone intervals were also observed in the lower part of the Hibernia Formation and in the upper part of the Johnson Formation (O'Brien and Woods 1995; O'Brien et al. 1999). Such stratigraphic relationships suggest that the Grebe Sandstone was interfingered with carbonate sediments during its deposition (Patillo and Nicholls 1990). Therefore the occurrence of thin limestone beds within the Grebe Sandstone is a reasonable scenario.

\section{GEOCHEMICAL RESULTS}

\subsection{Stable Isotopes}

The $\delta^{13} \mathrm{C}$ and $\delta^{18} \mathrm{O}$ composition of the carbonates from the Grebe Sandstone, the Hibernia Formation and the Johnson Formation are listed in Table 1. The cements in the calcareous sandstones of the Grebe Sandstone exhibit a nar-

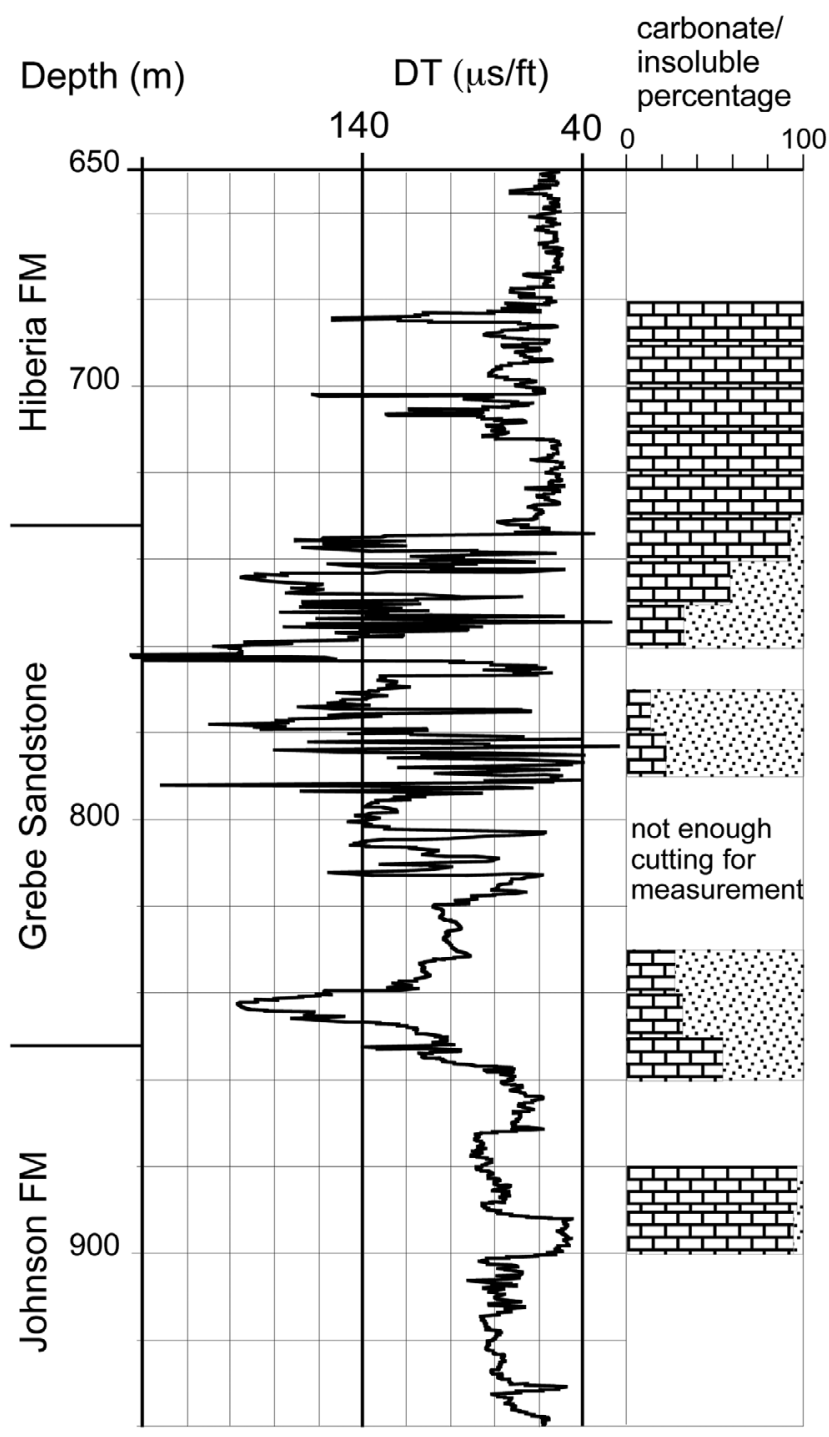

Fig. 4. Comparison between sonic log and carbonate/insoluble ratio of Jabiru-2 showing that the depth control of cuttings is reasonably good. Inverse of sound speed is presented in microsecond per feet. Greater values indicate slower speed, hence lesser cementation. 
Table 1. Results of mineralogical and geochemical analysis. Isotopes are expressed in \%o V-PDB, and Mn and Co concentrations in ppm.

\begin{tabular}{|c|c|c|c|c|c|c|c|c|}
\hline \multirow{2}{*}{ Well name } & \multirow{2}{*}{ Depth (m) } & \multirow{2}{*}{ Formation } & \multirow{2}{*}{ Sample type } & \multirow{2}{*}{ Mineralogy } & \multicolumn{2}{|c|}{ Isotopes } & \multirow{2}{*}{ Mn } & \multirow{2}{*}{ Co } \\
\hline & & & & & $\delta^{13} \mathrm{C}$ & $\delta^{18} O$ & & \\
\hline East Swan 2 & 1070 & Hibernia & lms-mic & $\mathrm{C}$ & -4.50 & -4.68 & 4.40 & $*$ \\
\hline East Swan 2 & 1090 & Hibernia & lms-mic & $\mathrm{C}$ & -4.53 & -4.63 & 4.99 & * \\
\hline East Swan 2 & 1110 & Hibernia & lms-mic & $\mathrm{C}$ & -4.14 & -4.54 & 7.59 & $*$ \\
\hline East Swan 2 & 1130 & Grebe & lms-mic & $\mathrm{C}$ & -4.03 & -4.78 & 9.58 & $*$ \\
\hline East Swan 2 & 1150 & Grebe & lms-mic & C 93\%, D 7\% & -6.80 & -4.76 & 7.12 & 0.10 \\
\hline East Swan 2 & 1150 & Grebe & sst-cmt & D $82 \%, C 18 \%$ & -6.67 & -1.77 & 20.59 & 0.19 \\
\hline East Swan 2 & 1170 & Grebe & 1ms-mic & $\mathrm{C}$ & -3.23 & -4.67 & - & - \\
\hline East Swan 2 & 1170 & Grebe & sst-cmt & D $67 \%$, C $33 \%$ & -11.21 & -2.44 & 25.89 & 0.17 \\
\hline East Swan 2 & 1190 & Grebe & sst-cmt & C $53 \%, \mathrm{D} 47 \%$ & -10.65 & -2.99 & 21.45 & 0.09 \\
\hline East Swan 2 & 1210 & Grebe & sst-cmt & D $55 \%, C 45 \%$ & -11.23 & -3.28 & 19.15 & 0.41 \\
\hline East Swan 2 & 1230 & Grebe & lms-mic & $\mathrm{C}$ & -5.57 & -4.97 & 5.49 & $*$ \\
\hline East Swan 2 & 1230 & Grebe & sst-cmt & C $64 \%$, D $36 \%$ & -19.52 & -4.01 & 14.59 & 1.17 \\
\hline East Swan 2 & 1270 & Grebe & lms-mic & $\mathrm{C}$ & -6.33 & -4.34 & - & - \\
\hline East Swan 2 & 1270 & Grebe & sst-cmt & $\mathrm{C}$ & -23.30 & -5.69 & 11.97 & 0.27 \\
\hline East Swan 2 & 1290 & Grebe & sst-cmt & $\mathrm{C}$ & -21.15 & -5.42 & 12.32 & 0.10 \\
\hline East Swan 2 & 1310 & Grebe & sst-cmt & C 67\%, D 33\% & -14.25 & -3.45 & 33.82 & 0.37 \\
\hline East Swan 2 & 1330 & Johnson & lms-mic & $\mathrm{C}$ & 0.28 & -2.75 & 21.64 & $*$ \\
\hline East Swan 2 & 1330 & Johnson & sst-cmt & C $90 \%$, D $10 \%$ & -6.01 & -2.95 & 55.47 & 0.54 \\
\hline East Swan 2 & 1350 & Johnson & lms-mic & $\mathrm{C}$ & -0.13 & -2.77 & 12.15 & $*$ \\
\hline East Swan 2 & 1350 & Johnson & sst-cmt & C $81 \%$, D $19 \%$ & -6.40 & -3.11 & 46.88 & 0.29 \\
\hline East Swan 2 & 1370 & Johnson & lms-mic & $\mathrm{C}$ & 0.40 & -2.94 & 13.82 & $*$ \\
\hline East Swan 2 & 1370 & Johnson & sst-cmt & $\mathrm{C}$ & 0.18 & -2.93 & 57.49 & 0.29 \\
\hline East Swan 2 & 1390 & Johnson & lms-mic & $\mathrm{C}$ & -3.35 & -2.85 & - & - \\
\hline East Swan 2 & 1390 & Johnson & sst-cmt & $\mathrm{C}$ & 1.19 & -2.64 & 49.58 & 0.10 \\
\hline East Swan 2 & 1410 & Johnson & 1ms-mic & $\mathrm{C}$ & 0.94 & -4.00 & 44.98 & 0.80 \\
\hline East Swan 2 & 1410 & Johnson & sst-cmt & $\mathrm{C}$ & 1.67 & -2.71 & - & - \\
\hline \multirow[t]{2}{*}{ Jabiru-2 } & 690 & Hibernia & lms-mic & C $81 \%$, D $19 \%$ & -5.90 & -4.07 & 8.23 & 0.11 \\
\hline & & & & & -5.79 & \multicolumn{2}{|c|}{-4.35 (repeat) } & \\
\hline Jabiru-2 & 700 & Hibernia & lms-mic & C $94 \%$, D $6 \%$ & -3.56 & -4.08 & 5.05 & 0.05 \\
\hline Jabiru-2 & 710 & Hibernia & lms-mic & C 97\%, D 3\% & -2.32 & -4.37 & 3.13 & $*$ \\
\hline Jabiru-2 & 720 & Hibernia & lms-mic & C 97\%, D 3\% & -2.00 & -4.71 & 3.53 & 0.05 \\
\hline Jabiru-2 & 730 & Hibernia & lms-mic & C $88 \%$, D $12 \%$ & -4.32 & -4.50 & 3.74 & 0.05 \\
\hline Jabiru-2 & 740 & Grebe & lms-mic & C 93\%, D 7\% & -3.35 & -4.70 & 6.58 & $*$ \\
\hline \multirow[t]{2}{*}{ Jabiru-2 } & 740 & Grebe & sst-cmt & C $92 \%$, D $8 \%$ & -19.63 & -5.23 & - & - \\
\hline & & & & & -19.33 & \multicolumn{2}{|c|}{-5.10 (repeat) } & \\
\hline Jabiru-2 & 750 & Grebe & lms-mic & C $88 \%$, D $12 \%$ & -3.94 & -5.66 & 8.53 & $*$ \\
\hline
\end{tabular}


Table 1. (Continued)

\begin{tabular}{|c|c|c|c|c|c|c|c|c|}
\hline \multirow{2}{*}{ Well name } & \multirow{2}{*}{ Depth (m) } & \multirow{2}{*}{ Formation } & \multirow{2}{*}{ Sample type } & \multirow{2}{*}{ Mineralogy } & \multicolumn{2}{|c|}{ Isotopes } & \multirow{2}{*}{ Mn } & \multirow{2}{*}{ Co } \\
\hline & & & & & $\delta^{13} \mathbf{C}$ & $\delta^{18} \mathrm{O}$ & & \\
\hline Jabiru-2 & 750 & Grebe & sst-cmt & C $69 \%$, D $31 \%$ & -23.21 & -3.51 & 37.01 & 0.59 \\
\hline Jabiru-2 & 760 & Grebe & lms-mic & C $91 \%$, D $9 \%$ & -5.03 & -4.48 & 8.91 & 0.06 \\
\hline Jabiru-2 & 760 & Grebe & sst-cmt & C $63 \%$, D $37 \%$ & -25.08 & -3.66 & - & - \\
\hline \multirow[t]{2}{*}{ Jabiru-2 } & 780 & Grebe & lms-mic & C $89 \%$, D $11 \%$ & -6.91 & -4.14 & 9.67 & 0.06 \\
\hline & & & & & -7.03 & \multicolumn{2}{|c|}{-3.90 (repeat) } & \\
\hline Jabiru-2 & 780 & Grebe & sst-cmt & C $82 \%$, D $18 \%$ & -27.31 & -3.83 & 52.73 & 1.58 \\
\hline Jabiru-2 & 790 & Grebe & lms-mic & C $74 \%$, D $26 \%$ & -10.22 & -3.51 & 10.03 & 0.06 \\
\hline Jabiru-2 & 790 & Grebe & sst-cmt & C $96 \%$, D $4 \%$ & -27.97 & -4.21 & 38.41 & 1.19 \\
\hline Jabiru-2 & 840 & Grebe & sst-cmt & D $76 \%$, C $24 \%$ & -19.58 & -1.48 & 47.45 & 1.19 \\
\hline Jabiru-2 & 850 & Grebe & lms-mic & C $98 \%$, D $2 \%$ & -3.59 & -4.05 & - & - \\
\hline Jabiru-2 & 860 & Grebe & lms-mic & $\mathrm{C}$ & -0.33 & -4.06 & - & - \\
\hline Jabiru-2 & 860 & Grebe & sst-cmt & D $87 \%$, C $13 \%$ & -17.87 & -1.10 & 49.73 & 0.90 \\
\hline Jabiru-2 & 890 & Johnson & lms-mic & C $88 \%$, D $12 \%$ & 1.43 & -3.73 & 11.07 & * \\
\hline \multirow[t]{2}{*}{ Jabiru-2 } & 890 & Johnson & sst-cmt & D $82 \%, C 18 \%$ & -15.89 & -1.15 & - & - \\
\hline & & & & & -16.17 & \multicolumn{2}{|c|}{-1.12 (repeat) } & \\
\hline Jabiru-2 & 900 & Johnson & $1 \mathrm{~ms}-\mathrm{mic}$ & C $80 \%$, D $20 \%$ & 1.02 & -3.64 & 12.29 & * \\
\hline Keeling-1 & 1370 & Hebernia & lms-mic & $\mathrm{C}$ & -0.49 & -4.25 & - & - \\
\hline Keeling-1 & 1370 & Hebernia & sst-cmt & C 93\%, D 7\% & -7.44 & -4.84 & 9.80 & 0.19 \\
\hline Keeling-1 & 1380 & Grebe & sst-cmt & $\mathrm{C}$ & -16.11 & -5.16 & 10.87 & 0.13 \\
\hline Keeling-1 & 1390 & Grebe & sst-cmt & C 97\%, D 3\% & -23.56 & -6.73 & 16.15 & 2.15 \\
\hline Keeling-1 & 1400 & Grebe & sst-cmt & $\mathrm{C}$ & -23.91 & -6.78 & 15.49 & 0.52 \\
\hline \multirow[t]{2}{*}{ Keeling-1 } & 1410 & Grebe & sst-cmt & $\mathrm{C}$ & -25.79 & -6.67 & 13.01 & 0.21 \\
\hline & & & & & -25.96 & \multicolumn{2}{|c|}{-6.54 (repeat) } & \\
\hline Keeling-1 & 1420 & Grebe & sst-cmt & $\mathrm{C}$ & -24.24 & -6.33 & 10.58 & 0.14 \\
\hline Keeling-1 & 1430 & Grebe & sst-cmt & $\mathrm{C}$ & -17.76 & -6.13 & 12.87 & 1.61 \\
\hline Keeling-1 & 1440 & Grebe & sst-cmt & $\mathrm{C}$ & -20.46 & -6.71 & 16.32 & 1.66 \\
\hline Keeling-1 & 1450 & Grebe & sst-cmt & $\mathrm{C}$ & -17.75 & -6.73 & 13.15 & 0.20 \\
\hline Keeling-1 & 1460 & Grebe & lms-mic & $\mathrm{C}$ & -2.07 & -5.01 & 0.67 & 0.67 \\
\hline \multirow[t]{2}{*}{ Keeling-1 } & 1460 & Grebe & sst-cmt & $\mathrm{C}$ & -16.91 & -6.65 & 12.87 & 0.17 \\
\hline & & & & & -17.06 & \multicolumn{2}{|c|}{-6.63 (repeat) } & \\
\hline Keeling-1 & 1480 & Grebe & lms-mic & $\mathrm{C}$ & -2.35 & -4.90 & 1.18 & * \\
\hline Keeling-1 & 1490 & Grebe & lms-mic & $\mathrm{C}$ & -3.02 & -4.90 & 2.36 & * \\
\hline Keeling-1 & 1500 & Grebe & lms-mic & C $52 \%$, D $48 \%$ & -0.81 & -4.38 & 3.61 & 0.05 \\
\hline \multirow[t]{2}{*}{ Keeling-1 } & 1510 & Grebe & lms-mic & $\mathrm{C}$ & -3.42 & -5.00 & 1.38 & 0.05 \\
\hline & & & & & -3.37 & \multicolumn{2}{|c|}{-4.95 (repeat) } & \\
\hline Keeling-1 & 1520 & Grebe & lms-mic & C $94 \%$, D $6 \%$ & -0.39 & -4.93 & 6.37 & 0.06 \\
\hline
\end{tabular}


Table 1. (Continued)

\begin{tabular}{|c|c|c|c|c|c|c|c|c|}
\hline \multirow{2}{*}{ Well name } & \multirow{2}{*}{ Depth (m) } & \multirow{2}{*}{ Formation } & \multirow{2}{*}{ Sample type } & \multirow{2}{*}{ Mineralogy } & \multicolumn{2}{|c|}{ Isotopes } & \multirow{2}{*}{ Mn } & \multirow{2}{*}{ Co } \\
\hline & & & & & $\delta^{13} \mathbf{C}$ & $\delta^{18} \mathrm{O}$ & & \\
\hline Keeling-1 & 1530 & Grebe & lms-mic & C $94 \%$, D $6 \%$ & -0.76 & -5.06 & 3.93 & * \\
\hline Keeling-1 & 1540 & Grebe & lms-mic & C 94\%, D $6 \%$ & -0.60 & -5.28 & 5.23 & * \\
\hline \multirow[t]{2}{*}{ Keeling-1 } & 1560 & Grebe & lms-mic & C $90 \%$, D $10 \%$ & -0.32 & -5.06 & 7.05 & * \\
\hline & & & & & -0.40 & \multicolumn{2}{|c|}{-5.01 (repeat) } & \\
\hline Keeling-1 & 1570 & Grebe & lms-mic & C 97\%, D 3\% & -0.85 & -5.41 & 5.00 & * \\
\hline Keeling-1 & 1580 & Grebe & lms-mic & $\mathrm{C}$ & -1.23 & -5.21 & 4.72 & * \\
\hline Keeling-1 & 1610 & Grebe & sst-cmt & C $76 \%$, D $24 \%$ & -14.00 & -6.91 & 24.40 & * \\
\hline \multirow[t]{2}{*}{ Keeling-1 } & 1620 & Grebe & sst-cmt & $\mathrm{C}$ & -13.69 & -6.73 & 18.18 & 0.18 \\
\hline & & & & & -13.49 & \multicolumn{2}{|c|}{-6.62 (repeat) } & \\
\hline Keeling-1 & 1630 & Grebe & sst-cmt & D $58 \%, C 42 \%$ & -3.81 & -3.96 & 36.50 & 0.56 \\
\hline Keeling-1 & 1640 & Grebe & lms-mic & C 95\%, D 5\% & -2.48 & -3.67 & - & - \\
\hline \multirow[t]{2}{*}{ Keeling-1 } & 1640 & Grebe & sst-cmt & C $58 \%$, D $42 \%$ & -3.80 & -3.87 & 33.63 & 0.50 \\
\hline & & & & & -3.98 & -3.99 & & \\
\hline \multirow[t]{2}{*}{ Keeling-1 } & 1650 & Grebe & sst-cmt & D $55 \%, C 45 \%$ & -9.83 & -5.12 & 37.58 & 0.40 \\
\hline & & & & & -9.65 & \multicolumn{2}{|c|}{-5.02 (repeat) } & \\
\hline Keeling-1 & 1660 & Grebe & sst-cmt & D $75 \%$, C $25 \%$ & -6.24 & -4.03 & 59.07 & 0.75 \\
\hline Keeling-1 & 1670 & Grebe & sst-cmt & D $72 \%$, C $28 \%$ & -6.88 & -4.07 & 57.62 & 0.65 \\
\hline Keeling-1 & 1680 & Grebe & sst-cmt & $\mathrm{C}$ & -1.77 & -3.81 & 104.59 & 2.80 \\
\hline Keeling-1 & 1690 & Grebe & sst-cmt & C $68 \%$, D $32 \%$ & -4.41 & -4.32 & 77.64 & 1.71 \\
\hline Keeling-1 & 1700 & Grebe & sst-cmt & C $74 \%$, D $26 \%$ & -0.38 & -4.76 & 110.69 & 2.18 \\
\hline \multirow[t]{2}{*}{ Keeling-1 } & 1710 & Johnson & sst-cmt & C $83 \%$, D $17 \%$ & 0.14 & -4.09 & 137.64 & 1.17 \\
\hline & & & & & 0.12 & \multicolumn{2}{|c|}{-4.09 (repeat) } & \\
\hline Keeling-1 & 1720 & Johnson & lms-mic & $\mathrm{C}$ & 1.16 & -3.69 & 66.08 & 0.22 \\
\hline Keeling-1 & 1720 & Johnson & sst-cmt & C 93\%, D 7\% & 0.67 & -3.19 & - & - \\
\hline Keeling-1 & 1730 & Johnson & lms-mic & $\mathrm{C}$ & 1.00 & -3.45 & 35.99 & 0.15 \\
\hline \multirow[t]{2}{*}{ Keeling-1 } & 1740 & Johnson & lms-mic & $\mathrm{C}$ & 0.98 & -2.87 & 18.24 & 0.17 \\
\hline & & & & & 0.99 & \multicolumn{2}{|c|}{-2.86 (repeat) } & \\
\hline Keeling-1 & 1750 & Johnson & lms-mic & $\mathrm{C}$ & 1.01 & -3.07 & - & - \\
\hline Keeling-1 & 1750 & Johnson & sst-cmt & $\mathrm{C}$ & 2.14 & -3.15 & 121.09 & 2.86 \\
\hline Keeling-1 & 1760 & Johnson & lms-mic & $\mathrm{C}$ & 1.35 & -2.82 & - & - \\
\hline Keeling-1 & 1760 & Johnson & sst-cmt & $\mathrm{C}$ & 2.50 & -2.86 & 103.54 & 1.77 \\
\hline Keeling-1 & 1770 & Johnson & lms-mic & $\mathrm{C}$ & 2.56 & -4.57 & 23.36 & 0.20 \\
\hline Keeling-1 & 1780 & Johnson & 1ms-mic & $\mathrm{C}$ & 0.78 & -5.94 & 15.64 & * \\
\hline \multirow[t]{2}{*}{ Keeling-1 } & 1790 & Johnson & lms-mic & C 95\%, D 5\% & 0.07 & -5.48 & 17.17 & * \\
\hline & & & & & 0.28 & \multicolumn{2}{|c|}{-5.40 (repeat) } & \\
\hline Keeling-1 & 1800 & Johnson & lms-mic & C $64 \%$, D $36 \%$ & 2.52 & -4.40 & 25.47 & 0.12 \\
\hline
\end{tabular}


Table 1. (Continued)

\begin{tabular}{|c|c|c|c|c|c|c|c|c|}
\hline \multirow{2}{*}{ Well name } & \multirow{2}{*}{ Depth (m) } & \multirow{2}{*}{ Formation } & \multirow{2}{*}{ Sample type } & \multirow{2}{*}{ Mineralogy } & \multicolumn{2}{|c|}{ Isotopes } & \multirow{2}{*}{ Mn } & \multirow{2}{*}{ Co } \\
\hline & & & & & $\delta^{13} \mathrm{C}$ & $\delta^{18} \mathrm{O}$ & & \\
\hline Skua-3 & 1210 & Hebernia & lms-mic & $\mathrm{C}$ & -1.53 & -4.23 & 1.69 & * \\
\hline Skua-3 & 1230 & Hebernia & lms-mic & $\mathrm{C}$ & -3.06 & -4.22 & 2.78 & 0.12 \\
\hline Skua-3 & 1260 & Grebe & lms-mic & $\mathrm{C}$ & -2.61 & -4.46 & 3.63 & 0.06 \\
\hline Skua-3 & 1290 & Grebe & sst-cmt & D $56 \%, C 44 \%$ & -15.21 & -3.69 & - & - \\
\hline \multirow[t]{2}{*}{ Skua-3 } & 1320 & Grebe & lms-mic & $\mathrm{C}$ & -3.59 & -4.82 & - & - \\
\hline & & & & & -3.60 & -4.80 & & \\
\hline \multirow[t]{2}{*}{ Skua-3 } & 1320 & Grebe & sst-cmt & $\mathrm{C}$ & -20.44 & -6.25 & 25.32 & 0.74 \\
\hline & & & & & -20.28 & -6.33 & & \\
\hline Skua-3 & 1330 & Grebe & $1 \mathrm{~ms}-\mathrm{mic}$ & $\mathrm{C}$ & -2.28 & -4.56 & - & - \\
\hline Skua-3 & 1330 & Grebe & sst-cmt & C $50 \%$, D $50 \%$ & -12.36 & -3.83 & - & - \\
\hline \multirow[t]{2}{*}{ Skua-3 } & 1340 & Grebe & sst-cmt & C $80 \%$, D $20 \%$ & -19.30 & -5.48 & 27.39 & 0.31 \\
\hline & & & & & -19.24 & -5.50 & & \\
\hline Skua-3 & 1350 & Grebe & sst-cmt & C $65 \%$, D $35 \%$ & -19.12 & -4.82 & 30.67 & 0.43 \\
\hline Skua-3 & 1360 & Grebe & sst-cmt & C $60 \%, \mathrm{D} 40 \%$ & -16.68 & -4.55 & 36.83 & 22.66 \\
\hline Skua-3 & 1370 & Grebe & sst-cmt & C $51 \%$, D $49 \%$ & -15.28 & -3.73 & 45.67 & 0.68 \\
\hline \multirow[t]{2}{*}{ Skua-3 } & 1380 & Grebe & sst-cmt & D $89 \%, C 11 \%$ & -8.55 & -1.86 & 80.12 & 1.93 \\
\hline & & & & & -8.60 & -1.80 & & \\
\hline Skua-3 & 1390 & Grebe & sst-cmt & D $96 \%, C 4 \%$ & -7.72 & -0.60 & 76.93 & 1.66 \\
\hline Skua-3 & 1400 & Grebe & sst-cmt & D $90 \%$, C $10 \%$ & -8.77 & -0.66 & 66.29 & 2.04 \\
\hline Skua-3 & 1410 & Johnson & lms-mic & C 97\%, D 3\% & -0.69 & -2.09 & 99.96 & 0.56 \\
\hline Skua-3 & 1420 & Johnson & lms-mic & C $98 \%$, D $2 \%$ & -0.14 & -2.15 & 89.44 & 0.26 \\
\hline Skua-3 & 1430 & Johnson & lms-mic & $\mathrm{C}$ & 0.42 & -2.82 & 23.55 & 0.06 \\
\hline Skua-3 & 1440 & Johnson & lms-mic & $\mathrm{C}$ & 0.75 & -2.67 & 9.05 & 0.12 \\
\hline Skua-3 & 1450 & Johnson & lms-mic & $\mathrm{C}$ & 0.91 & 0.91 & 9.61 & 0.12 \\
\hline Skua-3 & 1460 & Johnson & lms-mic & $\mathrm{C}$ & 0.56 & -2.83 & 13.07 & 0.06 \\
\hline
\end{tabular}

Note: Ims-mic: cements/matrix of limestone; sst-cmt: cements of calcareous sandstone; $\mathrm{C}:$ low-Mg calcite; D: dolomite; -: not enough reliable sample for analysis; *: below reliable detection limit (0.05 ppm).

(a)

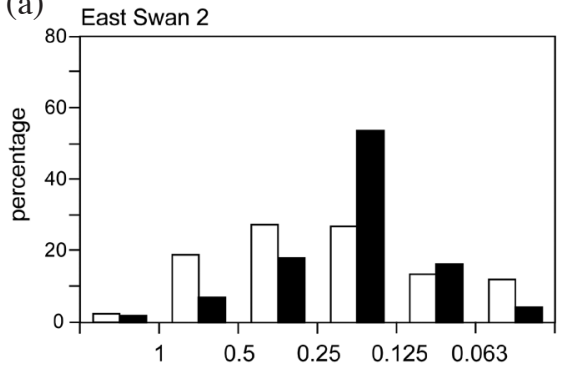

(b)

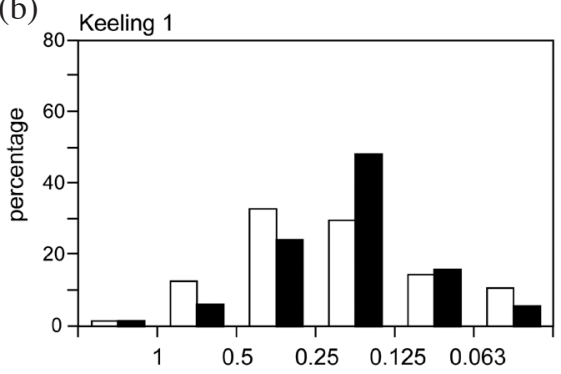

(c)

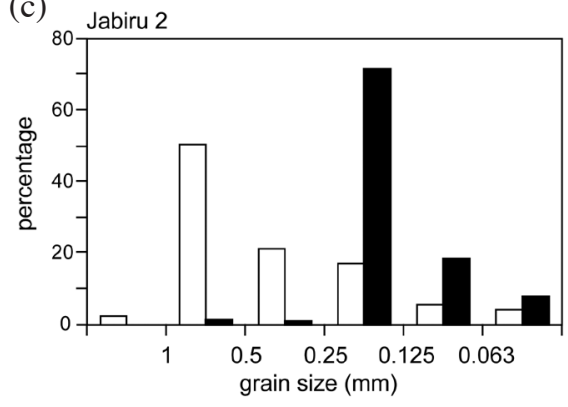

Legend: $\square$ total sands

sands in calcareous sandstones

Fig. 5. Comparisons between size distributions of the total quartz sands and the quartz sands in the calcareous cemented sandstones in the Grebe Sandstone of wells East Swan-2, Keeling-1 and Jabiru-2. Two size groups can be distinguished in the charts. Note the total sands include both the loose and cemented sands. 

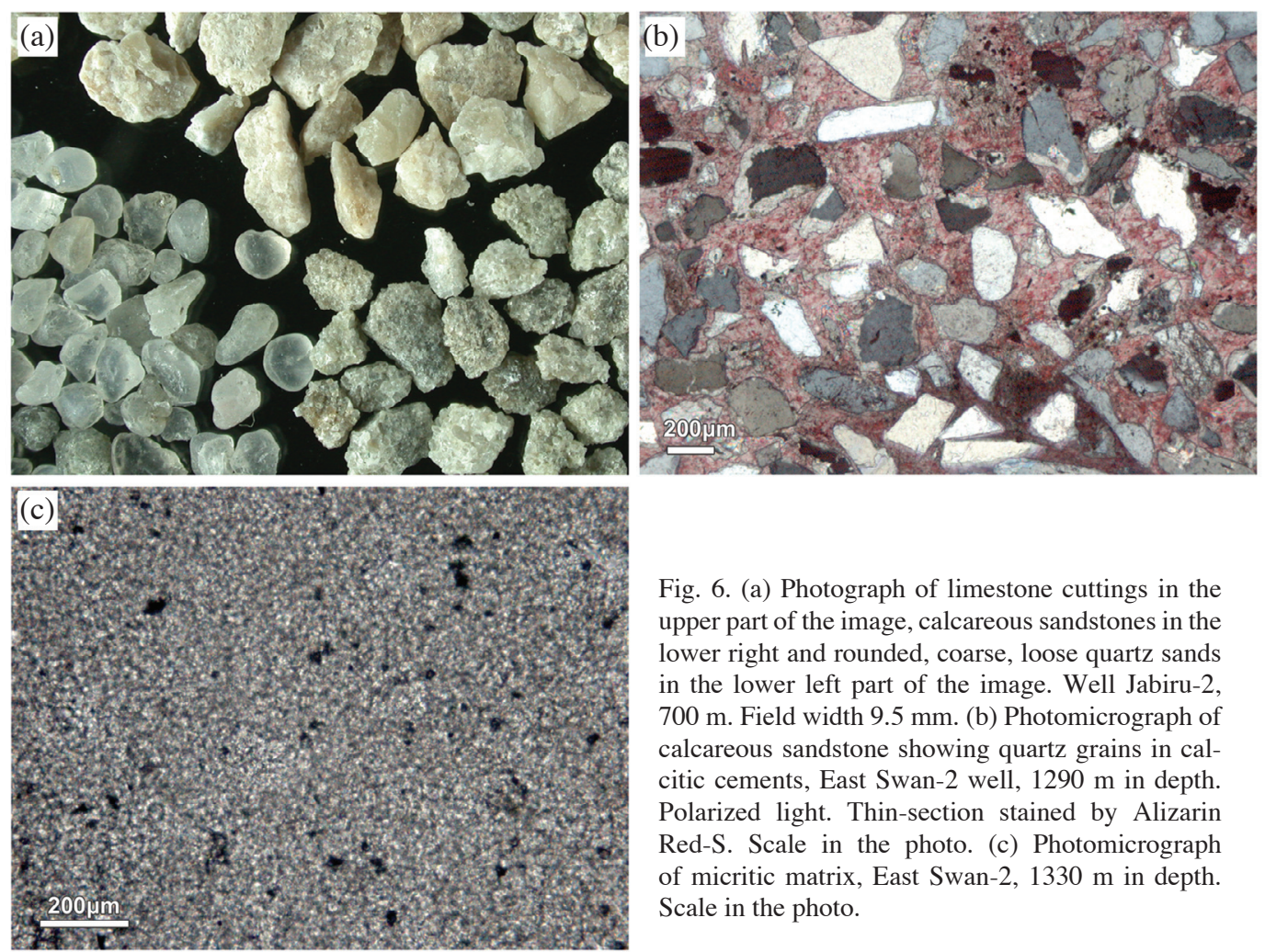

Fig. 6. (a) Photograph of limestone cuttings in the upper part of the image, calcareous sandstones in the lower right and rounded, coarse, loose quartz sands in the lower left part of the image. Well Jabiru-2, $700 \mathrm{~m}$. Field width $9.5 \mathrm{~mm}$. (b) Photomicrograph of calcareous sandstone showing quartz grains in calcitic cements, East Swan-2 well, $1290 \mathrm{~m}$ in depth. Polarized light. Thin-section stained by Alizarin Red-S. Scale in the photo. (c) Photomicrograph of micritic matrix, East Swan-2, $1330 \mathrm{~m}$ in depth. Scale in the photo.


Fig. 7. (a) SEM image of sparry calcite cement (S) and quartz grains (Qtz) in calcareous sandstone, with microspars (M) in the center of the pore space, well East Swan-2, 1190m. (b) SEM image showing calcite cements (C) and quartz grains (Q) in calcareous sandstone, well Keeling-1, 1370 $m$ in depth. (c) SEM image of the calcite cement (C) and quartz grain (Q) in calcareous, sandstone, well East Swan-2, $1230 \mathrm{~m}$ in depth. (d) SEM image of the micritic matrix in limestone, well East Swan-2, $1330 \mathrm{~m}$ in depth. 
row range in $\delta^{18} \mathrm{O}$ compositions from -6.91 to $-0.60 \%$ and a wide range in $\delta^{13} \mathrm{C}$ from -27.97 to $-0.38 \%$ (Fig. 8). On the other hand, most of micritic limestone grains from the Grebe Sandstone in all four wells show $\delta^{13} \mathrm{C}$ values from -6.97 to $-0.33 \%$ except for one datum at $-10.22 \%$, and even narrower $\delta^{18} \mathrm{O}$ composition from -5.66 to $-3.51 \%$, compared to those for the associated sandstone cements (Fig. 8).

The depletion in $\delta^{13} \mathrm{C}$ of sandstone cements can be better presented when samples from an adjacent depth in the same well are compared to each other. In the East Swan-2 well, the $\delta^{13} \mathrm{C}$ composition of sandstone cements of the Grebe Sandstone ranges from -23.30 to $-6.67 \%$, while the limestone grains were of -6.80 to $-3.23 \%$ (Fig. 9a). The $\delta^{13} \mathrm{C}$ composition of sandstone cements in the Jabiru-2 ranges from -27.97 to $-17.87 \%$, while those of the limestone grains range from -10.22 to $-0.33 \%$ (Fig. 9b). In the Keeling-1 well there are two intervals of sandstone. The $\delta^{13} \mathrm{C}$ composition of sandstone cements from 1380 to $1460 \mathrm{~m}$ in depth ranges from -25.88 to $-16.11 \%$, while those from the lower interval (1610 to $1700 \mathrm{~m}$ ) increase downward such that they are similar to the limestone grains with a $\delta^{13} \mathrm{C}$ value near $0 \%$ (Fig. 9c). The $\delta^{13} \mathrm{C}$ values of the sandstone cements in the Skua-3 well range from -20.36 to $-7.72 \%$, while the $\delta^{13} \mathrm{C}$ compositions of limestone matrix/cements are from -3.60 to $-2.28 \%$ (Fig. 9d).
The $\delta^{13} \mathrm{C}$ and mineral compositions of carbonate cements of the sandstones from Grebe Sandstone are plotted in Fig. 10. For Keeling-1, only the upper sandstone unit from 1380 to $1460 \mathrm{~m}$ in depth is included because only this interval shows a decrease in $\delta^{13} \mathrm{C}$. The plots show that the $\delta^{13} \mathrm{C}$ values generally become more positive with increasing dolomite content. The $\delta^{18} \mathrm{O}$ and mineral compositions of the sandstone cements are plotted in Fig. 11. Again, only samples from the upper sandstone unit are included. Similar to the pattern for $\delta^{13} \mathrm{C}$, the $\delta^{18} \mathrm{O}$ values generally become more positive with increasing dolomite content as well (Fig. 11).

\subsection{Trace Elements}

The results of the elemental analysis of the sandstone cements and limestones are listed in Table 1 and plotted in Fig. 12. Manganese concentrations range from about 10 to $138 \mathrm{ppm}$, and Co from about 0.1 to $20 \mathrm{ppm}$. Except for one sample from the Skua-3 well, $\mathrm{Mn}^{+2}$ and $\mathrm{Co}^{+2}$ contents show a roughly covariant relationship (Fig. 12). For comparative purposes, the $\mathrm{Mn}^{+2}$ and $\mathrm{Co}^{+2}$ concentrations of modern coldseep carbonates in the Gulf of Cadiz, Spain and the Pleistocene cold-seep carbonates of southwestern Taiwan are plotted together with our samples in Fig. 12 (Díaz-del-Río et al. 2003; Shih-Wei Wang, unpublished data).

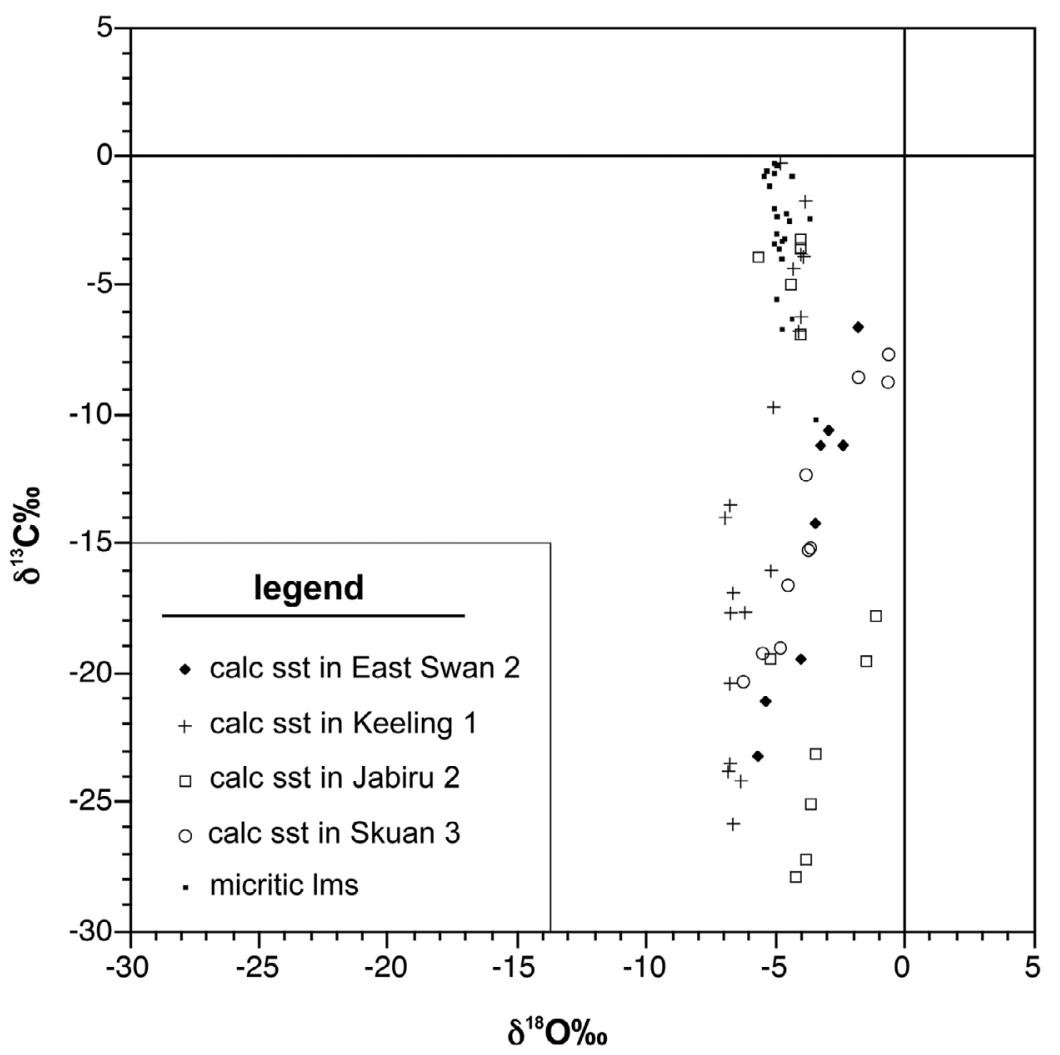

Fig. 8. Cross-plot showing stable carbon and oxygen isotopic composition of carbonate cements of the Grebe Sandstone in the four study wells Averaged values were plotted for repeated measurements of the same sample (Table 1). Note the narrow distribution of $\delta^{18} \mathrm{O}$ and wide distribution of $\delta^{13} \mathrm{C}$. See text for discussion. 
(a)

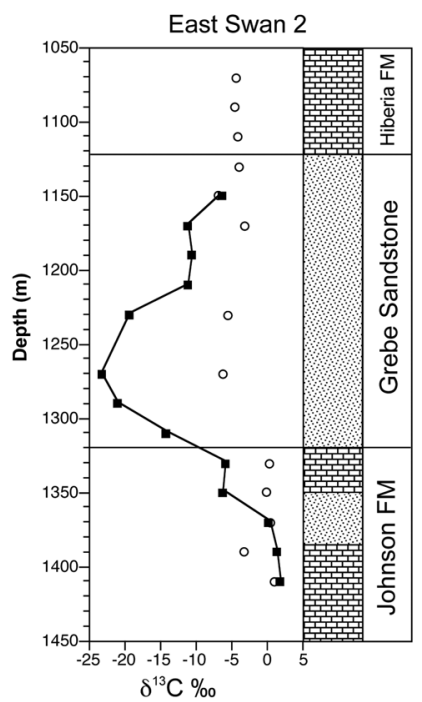

(b)

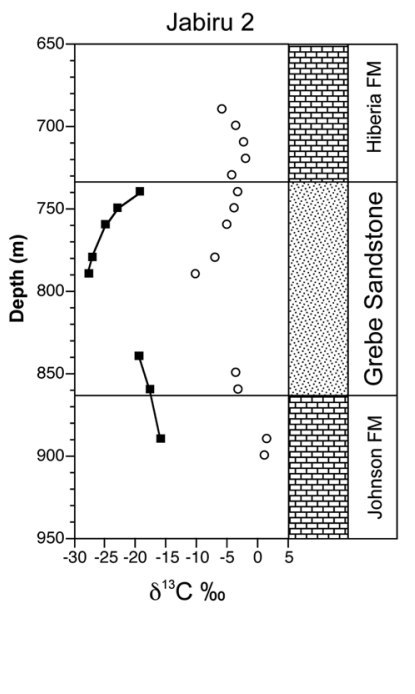

(c)

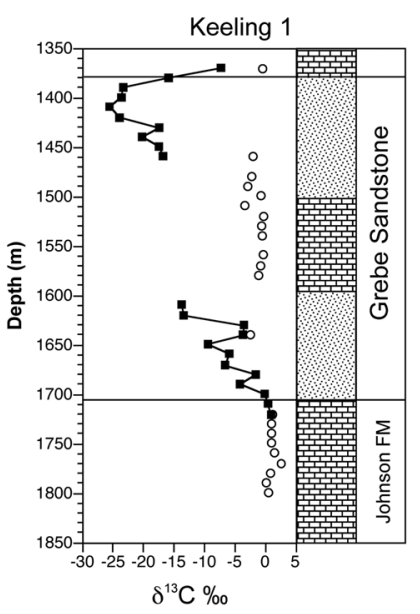

(d)

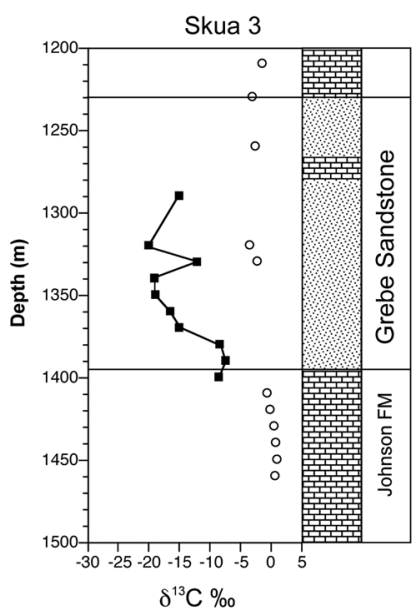

Fig. 9. Carbon isotope stratigraphy of the (a) East Swan-2 well; (b) Jabiru-2 well; (c) Keeling-1 well; (d) Skua-3 well. Solid square: cements of calcareous sandstone; open circle: matrix/cements of limestone. Averaged values were plotted for repeated measurements of the same sample (Table 1). Note the differences in $\delta^{13} \mathrm{C}$ values between sandstone cements and limestones. See text for discussion.

(a)

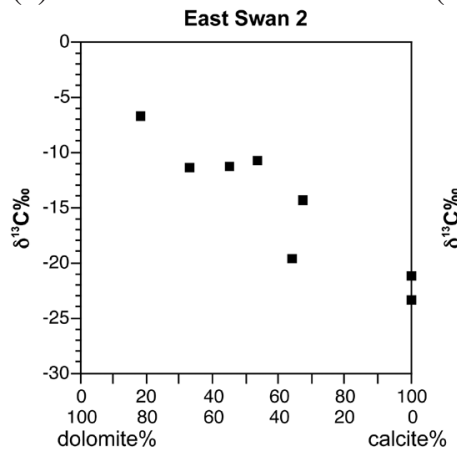

(b)

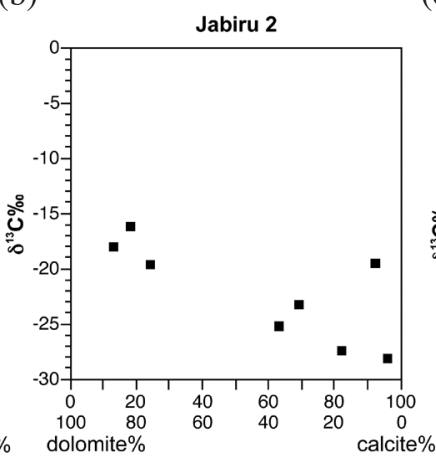

(c)

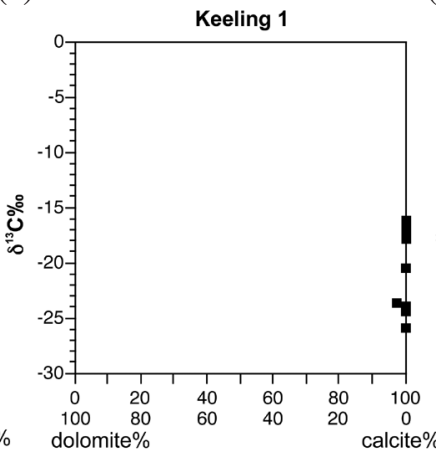

(d)

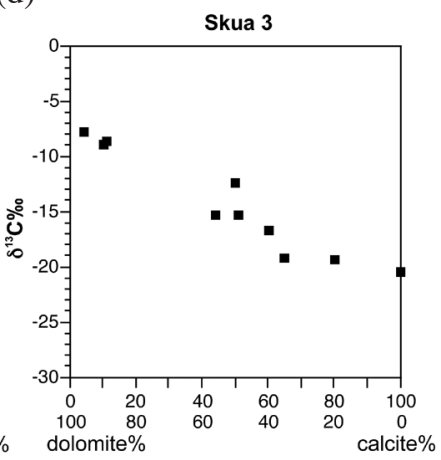

Fig. 10. Cross-plot showing $\delta^{13} \mathrm{C}$ and mineral compositions of sandstone cements in the Grebe Sandstone. $\delta^{13} \mathrm{C}$ generally decrease with increasing calcite abundance. For well Keeling-1, only the calcareous sandstones in the upper sandstone unit are included. Averaged values were plotted for repeated measurements of the same sample (Table 1). See text for discussion.

(a)

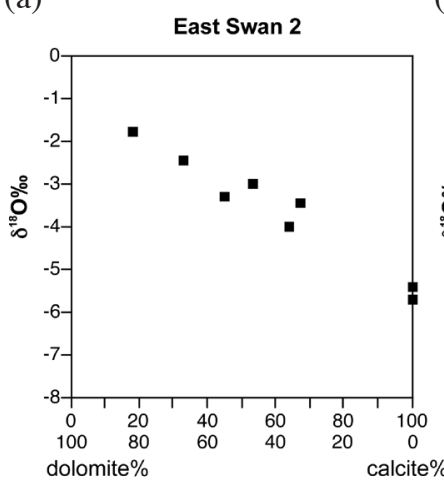

(b)

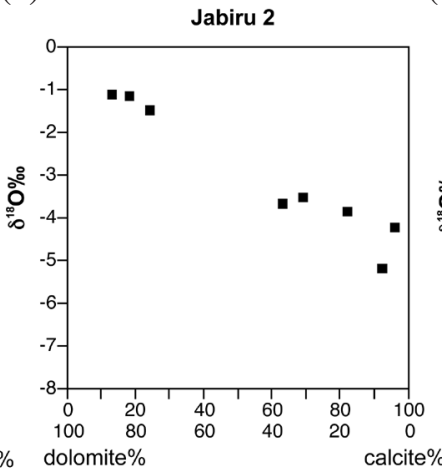

(c)

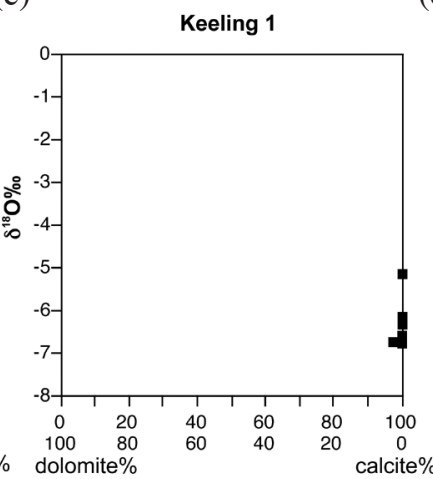

(d)

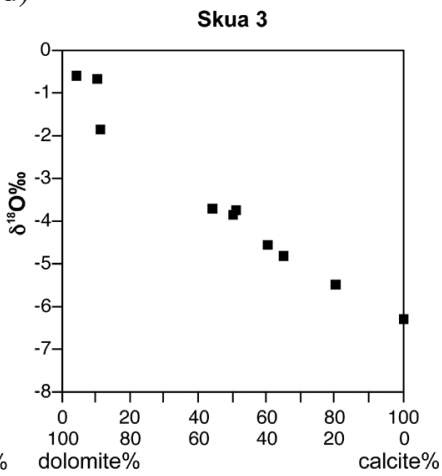

Fig. 11. Cross-plot showing $\delta^{18} \mathrm{O}$ and mineral compositions of sandstone cements of the Grebe Sandstone. $\delta^{18} \mathrm{O}$ generally decrease with increasing calcite abundance. For well Keeling-1, only the calcareous sandstones in the upper sandstone unit are included. Averaged values were plotted for repeated measurements of the same sample (Table 1). See text for discussion. 


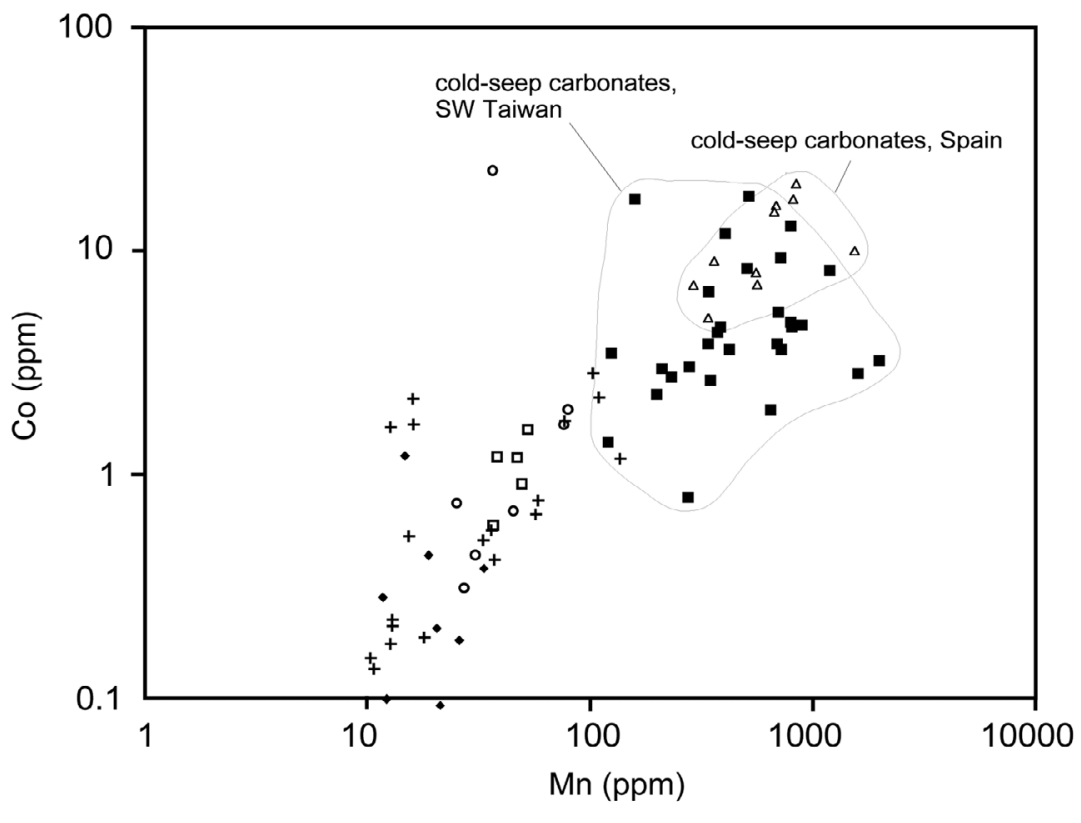

\section{Legend}

- Pleistocene cold-seep carbonate of SW Taiwan

$\triangle$ modern cold-seep carbonate offshore Spain

+ Keeling $1 \quad$ a Jabiru 2

- Skua 3 - East Swan 2

Fig. 12. Manganese and cobalt elemental compositions of sandstone cements in the Grebe Sandstone, compared to that of the Pleistocene coldseep carbonates of southwestern Taiwan (Shih-Wei Wang, unpublished data) and modern cold-seep carbonates of Spain (Díaz-del-Río et al. 2003, their Table 2). The two sites of cold-seep carbonate agree with each other in general, whereas the Grebe data are different from these two sites. See text for discussion.

\section{DISCUSSION}

\subsection{Hydrocarbon-Derived Carbonate Mineralization}

The stable isotope results show that the carbonate cements in sandstones and limestones from the Grebe Sandstone differ greatly in $\delta^{13} \mathrm{C}$ composition (Fig. 9). The $\delta^{13} \mathrm{C}$ values of the cements in limestone do not differ much from those in carbonates influenced by typical meteoric or burial diagenesis (Choquette and James 1990; James and Choquette 1990). $\delta^{13} \mathrm{C}$ values can be as low as about $-10 \%$ PDB in the case of meteoric diagenesis (James and Choquette 1990). Therefore, the $\delta^{13} \mathrm{C}$ compositions of the limestone matrix do not necessarily indicate a hydrocarbon-related origin.

On the other hand, the $\delta^{13} \mathrm{C}$ of the carbonate cements in sandstones ranges from about -28 to $0 \%$ (Fig. 8). Such a wide distribution cannot be explained by either meteoric or burial diagenesis. Meteoric diagenesis would result in $\delta^{13} \mathrm{C}$ values ranging from about -10 to 0\%o PDB (James and Choquette 1990), while burial diagenesis usually can only decrease the $\delta^{13} \mathrm{C}$ values by about $2 \%$, from about $2 \%$ o to approximately 0\% PDB (Choquette and James 1990). In other words, processes other than meteoric diagenesis or burial diagenesis must be involved to explain our data. We propose that such a wide range is caused by two factors. First, this is a result of the relative abundance of dolomite versus calcite in samples (See the following discussion). Second, it likely represents a mixture of carbon from two different sources. One source of carbon has $\delta^{13} \mathrm{C}$ values from about -7 to $0 \%$, similar to that of limestones from the Grebe Sandstone; other source of carbon shows $\delta^{13} \mathrm{C}$ values near -28\%o or less, considering that cuttings themselves represent a mixture over a $10-\mathrm{m}$ interval. A carbon source with a depleted $\delta^{13} \mathrm{C}$ composition near $-28 \%$ indicates a hydrocarbon-oxidation origin (Aharon 1994; Whiticar 1999; Campbell 2006), and is actually comparable to the $\delta^{13} \mathrm{C}$ values of well gases and oil in the Vulcan sub-basin where the $\delta^{13} \mathrm{C}$ values of methane is in the range of -43 to $-38 \%$, ethane from -30 to $-27 \%$ and saturated oil near -27\% PDB (O'Brien and Woods 1995, their Fig. 5).

Calcite and dolomite both occur in the calcareous sandstones with depleted $\delta^{13} \mathrm{C}$ values (Table 1). However, the relationship between $\delta^{13} \mathrm{C}$ compositions and relative abundance of the two minerals offer some hints regarding what was the primary hydrocarbon-derived mineral. Figure 10 shows that the lowest $\delta^{13} \mathrm{C}$ values always occur in completely calcitic cements, and the $\delta^{13} \mathrm{C}$ values generally 
increased with increasing dolomite abundance. This pattern is strong evidence that the isotopic value is dependent on relative abundance of calcite/dolomite, and that calcite is the primary mineral derived from hydrocarbon oxidation.

The dolomite, on the other hand, exhibits significantly higher $\delta^{13} \mathrm{C}$ values than the calcites. Only in Jabiru-2, a few dolomite-dominant samples have $\delta^{13} \mathrm{C}$ compositions ranging as negative as from -20 to $-16 \%$ (Fig. 12). Linear extrapolations suggest that $100 \%$ dolomitic samples of East Swan-2 and Skua-3 would have $\delta^{13} \mathrm{C}$ compositions about -4 and $-7 \%$ respectively, much less negative than the calcite-dominant samples (Fig. 12). These $\delta^{13} \mathrm{C}$ isotopic values can be easily explained by meteoric diagenesis of the precursor calcites. In addition, dolomite did not occur in the upper sandstone unit of the Grebe Sandstone in Keeling-1, where the $\delta^{13} \mathrm{C}$ values are much more negative than in the lower sandstone unit (Fig. 10). This is also an indication that calcite is the main resultant mineral of hydrocarbon-related cementation.

Changes in carbon isotopic compositions from precursor calcite to dolomite are negligible because of the very high rock/water ratios relative to carbon in pore water (Land 1992). The relatively higher $\delta^{13} \mathrm{C}$ values of the dolomitic samples are therefore interpreted to be a result of original $\delta^{13} \mathrm{C}$ compositions of the pore water in which the dolomites formed, but not from fractionation during dolomitization. It is thus concluded that, except for some dolomites in Jabiru-2, the majority of the dolomite cements in the Grebe Sandstone did not resulted from hydrocarbon oxidation but formed by dolomitization of precursor calcites, or from dolomite cementation in pore fluids, with $\delta^{13} \mathrm{C}$ values about -7 to $-4 \%$. Dolomitization or dolomite cements are very common in carbonates in burial environments (Mountjoy and Amthor 1994). It is natural to assume that these processes occurred in the Grebe Sandstone also. Dolomites of Jabiru-2 might have been partially derived from $\delta^{13} \mathrm{C}$-depleted calcites thus they exhibit lower $\delta^{13} \mathrm{C}$ values than other wells. The calcites of Jabiru-2 also show greater depletion in $\delta^{13} \mathrm{C}$ than other wells, implying more influences by hydrocarbon at the Jabiru-2 site than other sites.

\subsection{Sea-Floor or Subsurface?}

Authigenic carbonates have been found at many methane vents on the sea floor (Paull et al. 1984; Hovland et al. 1987, 1994; Roberts et al. 1989; Greinert et al. 2001; Campell 2006). This raises the question as to whether the localized carbonate cementation in the Grebe Sandstone actually occurred at sea-floor methane vents in the Eocene, which were then subsequently buried, as opposed to a shallow burial interpretation (O'Brein and Woods 1995).

Many studies have described petrographical features of carbonate crust at methane vents and these features are broadly similar to each other (e.g., Hovland et al. 1987; Ter- zi et al. 1994; Aloisi et al. 2000; Greinert et al. 2001; Peckmann et al. 2001; Campbell et al. 2002; Wang et al. 2006). The most common features are a dark gray, argillaceous, fine-grained carbonate matrix, carbonate breccias, fragments of specific mollusks such as vesicomyids or lucinids, siliciclastics, pelagic foraminifera, and botryoid or isopachous aragonitic cements. However, none of these features have been found in the Grebe Sandstone and its carbonate cements, which instead are generally light in colored, sparry crystals. It is thus unlikely that the localized carbonate cements in the Grebe Sandstone could have developed at seafloor methane seeps in the Eocene.

Figure 11 shows a range of $\delta^{18} \mathrm{O}$ values from -7 to $-1 \%$ and well-defined covariant relationships between $\delta^{18} \mathrm{O}$ composition and dolomite abundance in all wells except Keeling-1, in which dolomite is absent in the upper sandstone unit (see Fig. 9c and Table 1). We consider this covariant relationship to result from oxygen isotopic fractionation between calcite and dolomite and mixture of calcite and dolomite in samples. When calcite is dolomitized, $\delta^{18} \mathrm{O}$ composition of the replacive dolomite will be heavier than the precursor calcite by about 3.8\%o PDB (Land 1992). Considering that the samples contain mixed dolomite and calcite, and the fractionation in $\delta^{18} \mathrm{O}$ and ${ }^{16} \mathrm{O}$, the estimated $\delta^{18} \mathrm{O}$ values of $100 \%$ calcite should be about -7 to $-5 \%$ (Fig. $11)$. This is a very narrow range and rather more negative values compared to the $\delta^{18} \mathrm{O}$ compositions of Cenozoic to modern methane-seep carbonates at or near the sea floor, where $\delta^{18} \mathrm{O}$ values are found in a wide range from -3 to $>10 \%$ (Campbell et al. 2002).

Furthermore, as Fig. 12 shows, the $\mathrm{Mn}^{+2}$ and $\mathrm{Co}^{+2}$ concentrations of the sandstone cements of the Grebe Sandstone are significantly lower than those of the cold-seep carbonates from Spain and Taiwan, while the two cases of cold-seep carbonates are similar to each other. Mn concentrations in sedimentary calcite are generally considered to be controlled by redox condition and precipitation rate of the mineral (Brand and Veiser 1980; Mucci 1988) but very little is known about $\mathrm{Mn}$ in hydrocarbon-related carbonate. Details regarding Cobalt behavior in sedimentary carbonates are more limited. Nevertheless, the patterns shown in Fig. 12 support an origin that differs from authigenic carbonates at sea-floor methane vents. We therefore concur with the interpretation by O'Brien and Woods (1995) suggesting a shallow burial origin.

\subsection{Facies Control on the Occurrence of Hydrocarbon- Derived Carbonate Cements}

One of the most interesting characteristics of the cements is that in every study well the carbonate cementation has occurred preferentially in fine-grained, sub-angular sands (Figs. 4 and 5). In contrast, the associated coarsegrained, well-rounded sands are rarely cemented. This pat- 
tern suggests that the carbonate mineralization in the Grebe Sandstone is not only dependent on hydrocarbon leakage as O'Brien and Woods (1995) proposed, but also on the lithofacies of the host rocks.

At this point, we do not fully understand why the hydrocarbon-derived cementation in the Grebe Sandstone is facies dependent. It may be that the angular grain in the cemented fine sandstones provided more reaction sites for carbonate mineralization. It could also be a result of the lower permeability and smaller pore-space in the fine-grained sands, which may have been more favorable to habitation by methane-oxidizing and sulfate-reducing microbes.

This finding is potentially important to hydrocarbon exploration in the Vulcan Sub-basin and other areas. Some studies have used the extent of so-called HRDZ as a criterion to evaluate the hydrocarbon trap integrity in the Vulcan Sub-basin (O'Brien et al. 1998, 1999). A relatively large area of HRDZ was interpreted as an indication of greater hydrocarbon leakage, while a relatively small area of HRDZ was interpreted to be indicative of less leakage (O'Brien et al. 1998, 1999). However, if the occurrence of hydrocarbonderived cementation is also affected by the lithofacies of the host rocks, the extent of localized cementation in the Grebe Sandstone cannot be used as the sole criteria for evaluating trap integrity. Such hydrocarbon-derived cementation could be overrepresented where the fine-grained facies is dominant, or underrepresented where the coarse-grained facies is dominant. Therefore, the lithofacies of the host rock needs to be taken into consideration in addition to trap-integrity evaluation.

\section{CONCLUSIONS}

1. On the basis of petrography, stable isotopes and elemental geochemistry, we concur that the localized carbonate cementation in the Eocene Grebe Sandstone of the Vulcan Sub-basin was hydrocarbon-derived and occurred in a subsurface environment (O'Brien and Wood 1995; O'Brien et al. 1999).

2. Isotopic and mineralogical analyses indicate that only the calcitic cements in the calcareous sandstone facies of the Grebe Sandstone are related to hydrocarbon oxidation. However, co-existing dolomites in the calcareous sandstones were not hydrocarbon-derived, and neither were the micritic matrix of associated limestones.

3. The carbonate mineralization in the Grebe Sandstone was not only dependent on hydrocarbon leakage, but was also dependent on the lithofacies of the host rocks with a preferential occurrence in the fine-grained sandstones and rarely in the coarse-grained sandstones. Therefore, the extent of localized cementation in the Grebe Sandstone cannot be used as the sole criteria for evaluating trap integrity in the Vulcan Sub-basin.
Acknowledgements We thank the Chinese Petroleum Corporation (Taiwan) for providing this research opportunity. Thanks are given to Dr. Cheng Chang for his help with the SEM, also to Kun-Wei Chung, Shu-Chuan Chen, and GangLing Chang for their laboratory assistance. This study was supported by a research grant (93-F0108-3-2) from the Petroleum Fund of Ministry of Economic Affairs, and a grant (NSC94-2623-7-178-001-ET) from the National Science Council, ROC.

\section{REFERENCES}

Aharon, P., 1994: Geology and biology of modern and ancient submarine hydrocarbon seeps and vents: An introduction. Geo-Mar. Lett., 14, 69-73, doi: 10.1007/BF 01203716. [Link]

Aloisi, G., C. Pierre, J.-M. Rouchy, J.-P. Foucher, J. Woodside, and MEDINAUT Scientific Party, 2000: Methane-related authigenic carbonates of eastern Mediterranean Sea mud volcanoes and their possible relation to gas hydrate destabilisation. Earth Planet. Sci. Lett., 184, 321-338, doi: 10.1016/S0012-821X(00)00322-8. [Link]

Cadman, S. J. and P. R. Temple, 2004: Bonaparte Basin. Australia Petroleum Accumulations Report 5, $2^{\text {nd }}$ Edition, Geoscience Australia, Canberra.

Campbell, K. A., 2006: Hydrocarbon seep and hydrothermal vent paleoenvironments and paleontology: Past developments and future research directions. Palaeogeogr. Palaeoclimatol. Palaeoecol., 232, 362-407, doi: 10.1016/j.palaeo.2005.06.018. [Link]

Campbell, K. A., J. D. Farmer, and D. Des Marais, 2002: Ancient hydrocarbon seeps from the Mesozoic convergent margin of California: Carbonate geochemistry, fluids and palaeoenvironments. Geofluids, 2, 63-94, doi: 10.1046/j.1468-8123.2002.00022.x. [Link]

Choquette, P. W. and N. P. James, 1990: Diagenesis-9: Limestones - The burial diagenetic environment. In: McIlreath, I. A. and W. Morrow (Eds.), Diagenesis, Geosci. Can., Reprint Ser. 4, 75-111.

Cowley, R. and G. W. O'Brien, 2000: Identification and interpretation of leaking hydrocarbons using seismic data: A comparative montage of examples from the major fields in Australia's northwest shelf and Gippsland Basin. Aust. Petrol. Prod. Explor. Assoc. J., 40, 121-150.

Díaz-del-Río, V., L. Somoza, J. Martínez-Frias, M. P. Mata, A. Delgado, F. J. Hernandez-Molina, R. Lunar, J. A. Martín-Rubí, A. Maestro, M. C. Fernández-Puga, R. León, E. Llave, T. Medialdea, and J. T. Vázquez, 2003: Vast fields of hydrocarbon-derived carbonate chimneys related to the accretionary wedge/olistostrome of the Gulf of Cádiz. Mar. Geol., 195, 177-200, doi: 
10.1016/S0025-3227(02)00687-4. [Link]

Gartrell, A., Y. Zhang, M. Lisk, and D. Dewhurst, 2004: Fault intersections as critical hydrocarbon leakage zones: Integrated field study and numerical modeling of an example from the Timor Sea, Australia. Mar. Petrol. Geol., 21, 1165-1179, doi: 10.1016/j.marpetgeo.2004.08.001. [Link]

Greinert, J., G. Bohrmann, and E. Suess, 2001: Gas hydrateassociated carbonate and methane-venting at hydrate ridge: Classification, distribution, and origin of authigenic lithologies. In: Paull, C. K. and W. P. Dillon (Eds.), Natural Gas Hydrates: Occurrence, Distribution, and Detection, AGU, Washington, DC, 99-113.

Hovland, M., M. R. Talbot, H. Qvale, S. Olaussen, and L. Aasberg, 1987: Methane-related carbonate cements in pockmarks of the North Sea. J. Sediment. Res., 57, 881-892, doi: 10.1306/212F8C92-2B24-11D7-864800 0102C1865D. [Link]

Hovland, M., P. F. Croker, and M. Martin, 1994: Fault associated seabed mounds (carbonate knolls?) off western Ireland and north-west Australia. Mar. Petrol. Geol., 11, 232-246, doi: 10.1016/0264-8172(94)9009 9-X. [Link]

James, N. P. and P. W. Choquette, 1990: Diagenesis-9: Limestones - The meteoric diagenetic environment. In: McIlreath, I. A. and W. Morrow (Eds.), Diagenesis, Geosci. Can., Reprint Ser. 4, 35-73.

Land, L. S., 1992: The dolomite problem: Stable and radiogenic isotope clues. In: Clauer, N. and S. Chaudhuri (Eds.), Isotopic Signatures and Sedimentary Records, Spinger-Verlag, New York, 49-68.

McCrea, J. M., 1950: On the isotopic chemistry of carbonates and a paleotemperature scale. J. Chem. Phys., 18, 849-857, doi: 10.1063/1.1747785. [Link]

Mountjoy, E. W. and J. E. Amthor, 1994: Has burial dolomitization come of age? Some answers from the Western Canada Sedimentary Basin. In: Purser, B., M. Tucker, and D. Zenger (Eds.), Dolomites - A volume in Honour of Dolomieu. Int. Assoc. Sediment. Spec. Publ., 21, 203-229, doi: 10.1002/9781444304077.ch13. [Link]

O'Brien, G. W. and E. P. Woods, 1995: Hydrocarbonrelated diagenetic zones (HRDZs) in the Vulcan Subbasin, Timor Sea: Recognition and exploration implications. Aust. Petrol. Prod. Explor. Assoc. J., 35, 220252.

O'Brien, G. W., M. A. Etherige, J. B. Willcox, M. Morse, P. Symonds, C. Norman, and D. J. Needham, 1993: The structural architecture of the Timor Sea, northwestern Australia: Implication for basin development and hydrocarbon exploration. Aust. Petrol. Prod. Explor. Assoc. J., 33, 258-279.

O’Brien, G. W., M. Lisk, I. Duddy, P. J. Eadington, S. Cadman, and M. Fellows, 1996: Late Tertiary fluid migration in the Timor Sea: A key control on thermal and diagenetic histories. Aust. Petrol. Prod. Explor. Assoc. J., 36, 399-427.

O'Brien, G. W., P. Quaife, R. Cowley, M. Morse, D. Wilson, M. Fellow, and M., Lisk, 1998: Evaluating trap integrity in the Vulcan Sub-basin, Timor Sea, Australia, using integrated remote sensing geochemical technologies. In: Purcell, P. G. and R. P. Purcell (Eds.), The Sedimentary Basins of Western Australian, Petroleum Exploration Society of Australia, West Perth, 237-254.

O’Brien, G. W., M. Lisk, I. R. Duddy, J. Hamilton, P. Woods, and R. Cowley, 1999: Plate convergence, foreland development and fault reactivation: Primary controls on brine migration, thermal histories and trap breach in the Timor Sea, Australia. Mar. Petrol. Geol., 16, 533-560, doi: 10.1016/S0264-8172(98)000 70-1. [Link]

Osborne, M., 1990: The exploration and appraisal history of the Skua field, AC/P2 - Timor Sea. Aust. Petrol. Prod. Explor. Assoc. J., 30, 197-211.

Patillo, J. and P. J. Nicholls, 1990: A tectono-stratigraphic framework for the Vulcan Graben, Timor Sea region. Aust. Petrol. Prod. Explor. Assoc. J., 30, 27-51.

Paull, C. K., B. Hecker, C. Commeau, R. P. Feeman-Lynde, C. Neumann, W. P. Corso, S. Golubic, J. E. Hook, E. Sikes, and J. Curray, 1984: Biological communities at the Florida Escarpment resemble hydrothermal vent communities. Science, 226, 965-967, doi: 10.1126/science. 226.4677.965. [Link]

Peckmann, J., A. Reimer, U. Luth, C. Luth, B. T. Hansen, C. Henicke, J. Hoefs, and J. Reitner, 2001: Methanederived carbonates and authigenic pyrite from the northwestern Black Sea. Mar. Geol., 177, 129-150, doi: 10.1016/S0025-3227(01)00128-1. [Link]

Roberts, H. H., R. Sassen, R. Carney, and P. Aharon, 1989: ${ }^{13} \mathrm{C}$ depleted authigenic carbonate buildups from hydrocarbon seeps, Louisiana continental slope. Trans. Gulf Coast Assoc. Geol. Soc., 39, 523-530.

Terzi, C., P. Aharon, F. R. Lucchi, G. B. Vi, 1994: Petrography and stable isotope aspects of cold-vent activity imprinted on Miocene-age "calcari a Lucina" from Tuscon and Romagna Appenines, Italy. Geo-Mar. Lett., 14, 177-184, doi: 10.1007/BF01203729. [Link]

Wang, S. W., S. Y. Gong, H. S. Mii, and C. F. Dai, 2006: Cold-seep carbonate hardgrounds as the initial substrata of coral reef development in a siliciclastic paleoenvironment of southwestern Taiwan. Terr. Atmos. Ocean. Sci., 17, 405-427.

Whiticar, M. J., 1999: Carbon and hydrogen isotope systematics of bacterial formation and oxidation of methane. Chem. Geol., 161, 291-314, doi: 10.1016/S00092541(99)00092-3. [Link]

Woods, E. P., 1991: Eocene seismic velocity anomalies from the Timor Sea. Explor. Geophys., 22, 461-464. 